\title{
Récolte et mise en élevage d'Acariens oribates dans les conditions tchadiennes
}

\author{
par J. GRUVEL ef M. GRABER \\ Laboratoire de Farcha-Fort-Lamy, Tchad
}

\begin{abstract}
RÉSUMÉ
10 Récolte des Oribates.

La recherche des Oribates a été entreprise à partir de prélèvements de terre ef de feuilles mortes, effectués dans les terrains du Laboratoire de FARCHA.

Les Oribates sont extraits des prélèvements par la méthode des entonnoirs de Berlèse ef la méthode des lavages. Les résultats obtenus par ces deux méthodes sont comparables.

La rıchesse des prélèvements en Oribates est variable selon le lieu d'origine ; les meilleurs gisements ant ainsi été déterminés et seuls utilisés dans la suire des travaux. Les zones les plus riches sont celles centrées par un arbre ménageant une ombre constante et sous lequel croît une petite végétation herbacée. Les feuilles morles de NEEMES (Azodirachta indica) et la couche superficielle qu'elles recouvrent, constituent également de très bons gisements. Les migrations verticales des Oribates sont très limitées ef la profondeur où on les rencontre le plus fréquemment est de 20 à $25 \mathrm{~cm}$. Les déplacements horizontaux sont également faibles.
\end{abstract}

Quinze espèces d'Oribates réparties en dix genres ont été récoltées.

\section{$2^{\circ}$ Elevage des Oribates.}

Une grande partie des Oribates récoltés, est mise en élevage in vitro au Laboratoire et dans leur milieu naturel en les concentrant dans des bacs métalliques.

Au Laboratoire, une première série d'essais a donné des résultats médiocres et a permıs de meltre au point un procédé sımple et efficace. Les Oribates sont placés dans des boîtes de PETR! sur un milieu constıtué de terre prélevée dans les lieux d'orıgine. La terre est préalablement chauffée d̀ $180^{\circ}$ pendant 1 heure afin de défruire la flore mycélıenne et la microfaune du sol pouvant gêner le développemen1 des Oribates.

La nourriture est assurée par une rondelle de pomme de terre qui entretient en outre l'humidilé favorable à l'élevage; des excréments de moutons, très apprécıés des Orıbales, complètent le milieu nutritif.

La température de la pièce d'élevage est comprise entre $24^{\circ}$ et $28^{\circ} \mathrm{C}$.

Les élevages mis en route en novembre, se poursuivenl encore en mars. Des cycles de reproduction ont été observés.

L'infestation expérimentale des Oribates a pu se fare dans les boîtes de PETRI par addition d'excréments de moulons riches en œufs de Cestodes.

L'élevage en bacs a pour but de maıntenir dans ieur milieu nalurel el dans un volume réduit, une grande quantité d'Oribates.

Ces bacs sont fails en tôle, carrés $(1,20 \times 1,20 \mathrm{~m})$ sans fond et profonds de $0,70 \mathrm{~cm}$. IIs sont placés en terre de façon à dépasser la surface du sol de $10 \mathrm{~cm}$ environ.

Le contenu de ces bacs est enrıchi par l'appart d'Oribates prélevés dans les 
différents gisements; plus de 5.000 Oribates ont été ainsi.stockés dans chaque bac utilisé.

Cette méthode d'élevage permet de disposer constamment d'un grand nombre d'Oribates, de réaliser leur infestation massive par des œufs de Cestodes dont le cycle évolutif est à l'étude. Les Oribates alors infestés, constituent une réserve de parasites du mouton qui peut être utilısée pour foute étude parasitologique pratique : épidémiologique, thérapeutique.

Bibliographie : 17 références.

La recherche des Oribates a été entreprise dans le but d'étudier la transmission de Cestodes du mouton, à partir de prélèvements de terre et de feuilles mortes effectués dans les terrains du Laboratoire de FARCHA. Les différents points prospectés étant : des terrains ombragés par diverses espèces végétales (arbres ou plantes herbacées), des terrains piétinés par les animaux, des terrains nus, non fréquentés et jardins recouverts de feullies mortes.

\section{RÉCOLTES}

Les prélèvements consistent en mottes de terre de 2 décimètres cube, extraites chaque matın à différentes profondeurs.

L'extraction des Acariens est faite suivant deux méthodes : action de la chaleur et de la lumière (principe Berlèse) et lavage de la terre.

Batterie Berlèse : réalisée au Laboratoire par la mise en série de 6 entonnoirs chauffés par une lampe de $250 \mathrm{~W}$ placée au-dessus de chacun d'eux; l'extrémıté inférieure de l'entonnoir étant fermée par un tampon de coton humide. La motte de terre placée dans le cône de l'entonnoir est soumise à l'effer thermique de la lampe pendant 24 heures. Sous l'influence de la lumière, de la chaleur ef de la dessiccation produte, les Acartens présents dans la matte de terre migrent vers le bouchon de coton humide et s'y concentrent. Ils sont alors prélevés et triés. L'ensemble de l'appareil est maintenu dans la partie obscure du Laboratoire.

Méthode des lavages : la terre prélevée est immergée dans un seau d'eau ; après brassage destiné à débiter complètement la motte, Acariens et Insectes remontent à la surface de l'eau avec divers débris végétaux. L'ensemble constitue une mousse qui est recuellie avec une passoire ef placée dans des lignes pliées. Après séchage complet, on procède au tri de la faune ainsi isolée.
Les résultats obtenus par ces deux méthodes sont comparables seule l'orıgine du prélèvement est importante. Un passage de 24 heures dans la batterie est suffisant pour extraire toute la faune d'un prélèvement. Le lavage présente l'avantage de pouvoir traiter en une seule fois une plus grande quantité de terre.

26.657 Oribates appartenant à plusieurs espèces, ont été récoltés au cours de l'année 1963 et les deux premiers mois de 1964. La richesse des prélèvements est variable selon leur origine. Les melleures moyennes (quotient du nombre total d'Oribates récoltés en un lieu par le nombre de prélèvements correspondants) se limitent à quelques terrains qui seuls resteront exploités. Des prospections préalables ayant été faites en un grand nombre de points afin de déterminer les meilleurs gisements d̀ Oribates. Les meilleurs résultats correspondent aux origines suivantes : - les feuilles mortes, (à l'époque où elles existent, de novembre à mars) provenant des Neemes (Azadirachto indica) et la couche superficielle de terre qu'elles recouvrent, constituent les lieux les plus riches en Oribates:

- les arbres donnant le plus d'ombre et sous lesquels croît une abondante végétation basse détermınant un biotope favorable au développement des Oribates.

Cette flore entretient une ombre constante et retıent une humidité suffisante. Les conditions écologiques ainsi créées ne sont pas liées aux espèces végétales qui sonf très nombreuses et très variées, mais à la disposition de la végétation.

Vingt-cinq espèces ont été dénombrées à proximité des gisements à Oribates; les plus fréquentes sont :

Bolantes aegyptiaco (Zygophyllacées).

Tamarindus indica.

Diospiros mespiliformis (Ebenacées).

Azadirachio indica (Méliacées).

Diciptero sp. (Acanthacées). 
Leucas martınicensis (Labiées).

Hyptıs spicigera (Labiées).

Pulicaria undulata (Composées).

La présence d'arbres n'est cependant pas Indispensable ; un de nos terrains, régulièrement irrigué et portant une petite végétation, héberge constamment des Oribates.

Les terrains ensoleiliés sans végétation sont pratiquement dépourvus d'Oribates.

De plus, tous les prélèvements effectués à proximité de termitières ef des nids à fourmis ont toujours donné des récoltes plus abondantes. $\mathrm{Ce}$ fait s'explique par l'état du terrain qui est ameublé par les travaux de ces insectes sociaux ef facilite ainsi les déplacements ef l'installation des Acariens.

La profondeur optimum des prélèvements se situe en toutes saisons à $20-25 \mathrm{~cm}$. La migration verticale des Oribates dans le sol est de très faible amplitude. Au mament de la fraîcheur matinale, les Acariens se rencontrent plus nombreux près de la surface du sol et se concentrent à la profondeur de $20 \mathrm{~cm}$ dans le courant de la matinée.

En saison des pluies, ils peuvent descendre jusqu'à $40 \mathrm{~cm}$, nous n'en avons jamais trouvé plus profondément.

Les Oribates se localisent donc à un niveau où l'humidité convenable se maintient constante, fuyant la saturation en eau de la surface ou son dessèchement selon l'époque de l'année. Les déplacements horizontaux sont également très faibles.

D'autre part, des prélèvements de contrôle ont été effectués régulièrement dans les fourrages destinés à l'alimentation de moutons d'expérience. Prélèvements provenant :

- du foin tombé de la meule de stockage;

- des restes laıssés au fond des mangeoires:

- des fourrages verts récoltés quotidiennement.

Seul le foin de stockage en meule a donné quelques individus.

Les Oribates récoltés ont été soumis pour étude zoologique à Monsieur J. A. WALLWORK du Westfield College à Londres, qui a bien voulu assurer la détermination des espèces dont voici la liste:

Scheloribates perforotus n. $\mathrm{sp}$.

Scheloribotes conglobatus n. sp.

Scheloribotes fimbriatus Thor.
Unguizetes reticulatus $n$. sp.

Ceratozetes rostroserratus $\mathrm{n}$. $\mathrm{sp}$.

Zygoribatula setosa EVANS.

Africocorus colcorotus n. gen. n. sp.

Galumna baloghi n. sp. pour le Tchad.

Allogolumno pellucido n. sp.

Trichogalumna lunai BALOGH.

Trichogolumno microseta $\mathrm{n}$. sp.

Oppio fusıformis WALL. ssp. Iyroseta n. sp.

Oppia pilosella BALOGH ssp. longisera n. ssp.

Oppia heterosa n. sp.

Hypozetes translamellatus n. sp.

II est impossible pour le moment d'établir les proportions des différentes espèces récoltées ; celles appartenant au genre Scheloribates sont cependant les plus fréquentes.

\section{ÉLEVAGES DES ORIBATES}

Les Oribates récoltés ont été mis en élevage in vitro au Laboratoire et dans leur milieu naturel.

\section{Elevage au Laboratoire}

Les premiers essais d'élevage furent entrepris en cristallisoirs de $8 \mathrm{~cm}$ de diamètre sur $3,5 \mathrm{~cm}$ de profondeur, recouverts d'une gaze moustiquăıre fixée par un élastique. Le milieu d'élevage étant constitué par un fond de papier filtre sur lequel était répandu un peu de terre humide prélevée sur les lieux de récolte. Les Oribates triés étaient introduits ensulte à l'aide d'un pinceau humide, et l'ensemble placé dans une armoire climatisée d̀ $25^{\circ}$ avec une humidité de 95 p. 100 . Cınq pots ont été ainsi mis en observalıons et examınés chaque jour; les survies ont été respectivement de 17 jours, 11 jours, 9 jours, 10 jours, 8 jours. La fin de l'élevage était précisée par l'absence de tout Oribate vivant dans le milieu. D'une manière générale, la population dımınue très rapidement les premiers jours. se maintient pendant quelques jours et décroît ensuite très rapidement jusqu'à la fin.

La méthode utilısée dans cette première série d'expériences a donné des résultats médiocres; I'humidification du milieu faite chaque jour permettait la formation rapide d'un abondant feutrage de moisissures qui, favorable au début, étouffait rapidement les Oribates. De plus, quelques-uns d'entre eux parvenaient à s'échapper. 
A partir du mois de novembre, un autre procéđé d'élevage a été essayé et a donné satisfaction. Il $a$ en outre été facilité par la température ambiante qui s'est maintenue pendant plusieurs mois entre $24^{\circ}$ et $28^{\circ} \mathrm{C}$ dans la salle de travail. Dans cette $2^{\mathrm{e}}$ série d'expériences, le milieu d'élevage a été placé en boîtes de PETRI, constitué par de la terre chauffée d $180^{\circ}$ pendant une heure, afin de détruire la flore mycélienne ef la microfaune du sol. Le milieu nutritif étant constitué par des rondelles de pomme de terre qui assurent en outre le maintien d'une hygrométrie favorable ef constante. L'introduction d'excréments de mouton dans chaque boîte est également bénéfique, et permet en outre de réaliser l'infestation expérimentale des Oribates par des œufs de Cestodes du mouton.

Les Oribates, mis en élevage dans chaque boîte ainsi préparée, vivent encore 5 mois après : certaines espèces ayant donné deux cycles de reproduction.

Une meilleure survie est obtenue à partir d'Oribates extraits par lavage plutôt que par passage dans les entonnoirs desséchants.

\section{ÉLEVAGE DANS LE MILIEU NATUREL : BACS DE STOCKAGE}

Dans le but de conserver des populations d'Oribates importantes dans un volume réduit, 5 bacs d'élevage ont été placés sous les arbres les plus favorables à leur maintien. Ces bacs, en tôle, sans fond, d̀ section carrée $(1,20 \times 1,20 \mathrm{~m})$, profonds de $0,70 \mathrm{~m}$, sont enfoncés dans le sol de façon à dépasser sa surface de $10 \mathrm{~cm}$ environ. Les Acariens y sont maintenus prisonniers, ne pouvant s'échapper par le bas en raison de leurs migrations verticales réduites.

L'enrichissement de ces bacs a été fait à partir de récoltes d'Oribates effectuées aux environs; plus de 5.000 Oribates ont été ainsi stockés dans chaque bac utilisé deux fois par semaine.

Cette méthode d'élevage permet de disposer constamment d'un grand nombre d'Oribates, de réaliser leur infestation massive par des œufs de Cestodes dont le cycle évolutif est à l'étude. Les Oribates alors infestés constituent une réserve de parasites du mouton qui peut-être utilisée pour toute étude parasitologique pratique : épidémiologique ou thérapeutique.

\section{SUMMARY}

Collection and breeding of oribatids inites in Chad

\section{$1^{0}$ Collection of the Oribatids.}

Research on Oribatids has been started from samples of earth and dead leaves taken from the grounds of the laboratory of FARCHA.

The mites are isolated from the samples either by the system of Berlèse funnels or by washing. The results obtained by the two methods are comparable.

The content of mites in the sample varies according to place of origine : the best sources have thus been found and alone used from then on. These were found to be areas such as those around a tree giving a consiant shade and under which grows a herbaceous vegetation. The dead leaves of Azadirachta indica and the superficial layer that they cover are equally good sources. Oribads migrate vertically, only a very short way and one finds them most often at a depth of $20-25 \mathrm{~cm}$. Neither horizontally so they move very much.

Fifteen species of Oribatids of ten genera have been collected.

\section{$2^{\circ}$ Propagation of the Oribatids.}

One large part of the collected mites is kept in vitro'at the laboratory in their natural habitat, out closed in metal containers.

At the laboratory, a first serıes of trials has given moderate results which has mode it possible to outline a simple and effective procedure. The mites are put in PETRI dishes on a medium cansisting of earth taken from their place of origin. The earth has been previonsly heated to $180^{\circ}$ for one hour to destroy the fungal flora and the microfauna of the soil which could hinder the development of the mites. 
Nourishment is assured by a slice of potato which besides provides a moisture favorable for propagation ; sheep faeces, very favorable to Oribatıds, completes the nutritive medium.

The temperature of the room is maintained between $24^{\circ}$ and $28^{\circ} \mathrm{C}$.

The cultures, started in November, were still continuing in March and the reproductive cycles have been observed.

The experimental infection of Oribatıds has been possible in the PETRI dishes by the addition of sheep faeces containing many Cestode eggs.

The culture in containers was in order lo maintain a large quantity of mites in their natural environment but in a reduced volume. These confainers are of steel, square $(1,20 \times 1,20 \mathrm{~m})$ without boltom and of a depth of $10 \mathrm{~cm}$. They are placed in the soil so that they project about $10 \mathrm{~cm}$ above the ground.

The contents of the contaners is concentrated by putting in Oribatids taken from various sources. More than 5.000 Oribatids have been thus stocked in each container.

This methed of culture provides a large number of mites constantly af disposal and alse permits a massive infection of them by Cestode eggs, the life cycle of which is to be studied. Oribatids thus infected constitute a reserve of Cestodes which may be used for all practical parasıtological studies whether epidemiological or therapeutic.

Bibliography : 17 references.

\section{RESUMEN}

\section{La recolección y la crianza de acáridos Oribatidos en las condiciones de la región del Tchad}

\section{La recolección de los Oribatidos.}

La busqueda de los Oribatos se emprendio a partir de muestras de tierra y de hojas secas, recogidas en los terrenos del Laboratorio de FARCHA.

Se extraen los Oribatos de las muestras mediante el método de los embudos de Berlèse y el método de los lavados. Los resultados abtenıdos con estos dos métodos pueden ser comparados.

La riqueza de las muestras, en cuento a los Oribatos, varı según la locahidad de origen. De este modo fueron determinadcs los mejores yacimientos, los cuales se emplearon en los trabajos siguientes. Las zonas más ricas son las centradas por un arból dando una sombra constante $y$ debojo del cual crece una pequeña vegetación herbócea. Las hojas secas de Neemes (Azadirachta indica) y la capa superficial que estas cubren constituyen lambién muy buenos yacimientos. Las emigraciones verticales de los Oribalos son muy limitadas y es de 20 a $25 \mathrm{~cm}$. la profundidad en la que se les encuentra más frecuentemente. Son también pcco númerosos los traslados horizontales.

Fueron recolectadas quince especies de Oribatos distribuidas en diez generos.

$2^{\circ}$ Crianza de los Oribatos.

Una gran cantidad de los Oribalos recolectados son criados in vitro en el Laboratorio y en su ambiente natural concentrandolos en abrevaderos metálicos.

En el Laboratorio, una prımera serie de ensayos dió resultados mediocres $y$ permitió definir un proceso simple y eficaz. Los Oribatos son puestos en placas de PETRI sobre un medio constituido por tierra extraida en las localidades de arigen. Se ccienla previamente a $180^{\circ}$ durarite ına hora para destruir la flora miceliana y la microfouna del suelo que puede impedir el desarollo de los Oribatos

Se asegura la alimentación cor una rodaja de patata que además mantiene la humedad favorable para la crianza : las heces de ovejas, muy apreciadas por los Oribatos, completan el medio nutritivo. 
La temperaıura de la sala de crianza es de $24^{\circ}$ y $28^{\circ} \mathrm{C}$.

Las crianzas empezadas en noviembre, se prosiguen todavia en marzo. Se observaron ciclos de reproducción.

Se produjo una infección experimental de los Oribatos en las placas de PETRI con la adición de heces de ovejas ricas en huevos de céstodos.

La crianza en abrevaderos tiene por objeto mantener en su media natural y en un volumen reducido una gran cantidad de Oribatos.

Estos abrevaderos estan hechos con palastro, cuadrados $(1,20 \times 1,20 \mathrm{~m}) \mathrm{sin}$ fondo y profundos de $0,70 \mathrm{~cm}$. Se les caloca en tierra de tal modo que sobrepasen la superficie del suelo de cerca de $10 \mathrm{~cm}$.

La capacidad de estos abrevaderos esta enriquecida con el aporte de Oribatos expelidos en los diferentes yacimientos ; Fueron conservados más de 5000 Oribatos de esia manera en cada abrevadero empleado.

Este método de crianza permite de disponer constantemente de un gran número de Oribatos, de realizar la infección importante de ellos con huevos de céstodos cuyo ciclo de evolución se estudia. Los Oribatos infectados entonces, constituyen una reserva de parásitos de las ovejas que se puede utilizar para cualquier estudio parasitológico práctico : Epidemiologica, terapéutica.

Bibliografía : 17 referencias.

\section{BIBLIOGRAPHIE}

1. BOCZEK (J.). — The rearing method of small Insects and mites in controlled conditions of aire hymidity. Ekol. Polska. T. 2. Zoczyt, 1954, 4 : 473-6.

2. BOULANOVA-ZACHVATRINA (E. M.). Types écologiques de tiques à carapace et leur répartition dans le sol. Vie zoologique, 1952, 31.

3. GRANDJEAN (F.). - Sur l'élevage de certains Oribates en vue d'oblenir des clones. Bull. Mus. Nat. Hist. Nat. Paris, 1948, 2 (20) (5) : 450-7.

4. KATES (K. C.) et RUNKEL (C. E.). - Observations on Oribatid mites vectors of « MOniezia expansa » on pastures with a report of several new vectors from the United States. The Helminth. Soc. Wash. O. C., 1948, I5: 10-33.

5. KRULL $\left(W . H_{1}\right)$, 一 Observations on the distribution and ecology of the Oribatid mites. J. Wash. Acad. Scl., 1939, 29 (12) : 519-28.

6. KUZNETSON (M. J.). - The intermediate hosts of « Thysaniezia » and Avitellina infections in sheep. Veterinaryia, 1962, 39 (7) : 46-7.

7. MOREL (P.). - Les Cestodes du mouton. Thèse vétérinaire, Paris 1953.

8. PAULY (F.). - Zur Biologie einiger Belbiden und zur Funktion ihrer Pseudostigmatischen organe. Zool. Jahrb. Abt. f. Systen. ókol. und. Geog. der Tier., 1956, 84, 275-328.

9. RHODE (C. J.). - A modification of the plaster. Charcoal technique for rearing of mites and other small arthropods. Ecology, 1956. $37: 843-4$.

10. RIHA (G.). - Zin ökologie der Oribatiden in Kalksteinböden. Zool. Jadib. Abt. of. syst. ökol. u. Geog. du Tın. 1951, 80: 407-50.

11. SENGBUSH (H. S.). - Studies on the life history of three Oribatid mites with observations on other species. Ann. Ent. Soc. Amer., 1954, 47 : 646-667.

12. SITNIKOVA (L. G.)._-Sur la faune des tiques à carapaces (Acariformes, Oribatei) de la province du Riazan. Inst. Zool. de l'Acad. Sa. de I'U. R. S. S., 1958, 18, 163-75.

13. SITNIKOVA (L. G.). - Life cycles of some Oribatei and methods of culture. Zool. Zhur., 1959, 38 (11) : 1663-73.

14. SOLDAVOTA (A. P.). - Contribution to the study of the biology of Oribatid mites. R. AC. Sci. U. R. S. S., 1945, 46 (8) : 343-4.

15. STUNKARD $(H . W$.$) . - Studies on the life$ history of the Oribatid mites. Anat. Rec., 1944, $89: 550$.

16. WILLMANN (C.), - Moosbilben oder Oribatiden (Cryptostigmata). Die Tinwelt Deutschlands, 1931, $22: 79-200$.

17. WOODRING (J. P.) et COOK (E. F.). The biology of Ceratozetes, cesalpinus Berl., Schelotibates laevigatus K., Oppia neerlandica Oudms ; with "a description of all stages. Acarologia, 1962, 4 (1) : 101-37. 